REVISTA DE DERECHO UNED, NÚM. 11, 2012

\title{
LA PLAUSIBILIDAD CONSTITUCIONAL EN EL MARCO DEL EURO: ¿UNA CUESTIÓN EXCLUSIVAMENTE ECONÓMICA?
}

\author{
THE PLAUSIBILITY OF THE SPANISH CONSTITUTION \\ WITHIN THE EURO. \\ A PURELY ECONOMIC ISSUE?
}

\begin{abstract}
EDUARDo SANZ ARCEGA
Doctorando en Derecho Constitucional y en Economía Pública Universidad de Zaragoza
\end{abstract}

\begin{abstract}
Resumen: El crecimiento económico y unas finanzas públicas robustas y saneadas, finalidad a la que sirve la instauración de reglas fiscales, constituyen la condición indispensable para una plausible dotación de recursos económicos que, de forma efectiva, pueda materializar los postulados constitucionales. Sobre la premisa anterior, este trabajo tiene como objetivo presentar las diferencias económico-constitucionales entre el escenario pre-euro y las implicaciones acaecidas fruto de la integración de España en el euro. Para ello, se analiza la convivencia entre el Estado social y las dos crisis económicas más profundas ocurridas en nuestro país desde la instauración de la democracia hasta la adhesión a la Moneda Única. Acto seguido, se detalla la presente recesión dentro del contexto euro, en el que la soberanía sobre los principales instrumentos económicos de estabilización ya no reside en los Estados de la Eurozona. Finalmente, y dentro del presente marco económico-constitucional, se esbozan algunas propuestas de reforma encaminadas al fortalecimiento del Estado social, fórmula política en la que se constituye España.
\end{abstract}

Palabras clave: Estado social, Constitución, Crisis, Euro, España, Unión Europea. 
Abstract: Economic growth and robust public finances (aim of the introduction of fiscal rules) constitute the indispensable condition for a feasible and effective implementation of constitutional mandates. On this premise, this paper aims to present economic and constitutional differences between the pre-euro scenario and the implications of the adherence of Spain to the euro. To do this, we analyze the coexistence between the welfare state and the two deepest economic crises that have occurred in our country since the establishment of democracy and the adherence to the euro. Then, we detail the current recession in the European context, in which sovereignty over major economic stabilization instruments no longer resides in the States of the Eurozone. Finally, within this economic and constitutional framework, we explain some proposals of reform aimed at strengthening the Spanish Social State, the political formula in which Spain is constituted.

Keywords: Welfare State, Constitution, Crisis, Euro, Spain, European Union.

Recepción original: 06/11/2012

Aceptación original: 13/11/2012

Sumario: I. Introducción: España, Estado social federal; II. Crisis y ejercicio de soberanía económica (1977-1992); III. El euro y su primera crisis: el nuevo marco de la plausibilidad constitucional; IV. Conclusiones: ¿soberanos o intervenidos?

\section{INTRODUCCIÓN: ESPAÑA, ESTADO SOCIAL FEDERAL}

El texto constitucional del setenta y ocho, el primero en la Historia de España «obra corresponsable de la gran mayoría de las fuerzas políticas y no de una de ellas contra las demás» ${ }^{1}$, inauguraba un Estado social en el seno de la economía de mercado como organización socioeconómica y, correlativamente, la posibilidad, materializada a comienzos del siglo $\mathrm{XXI}^{2}$, de una descentralización

\footnotetext{
${ }^{1}$ SOLE TURA, Jordi, Nacionalidades y nacionalismos en España, Alianza, Madrid, 1985, 233 págs., pág. 83.

${ }^{2}$ Así, si bien cada Estado políticamente descentralizado ofrece caracterítiscas singulares (valga como ejemplo BLANCO VALDÉS, Roberto Luis, Los rostros del federalismo, Madrid, Alianza, 2012, 408 págs.), como principales elementos federales, observables en el entramado institucional español, resultan la coexistencia de dos niveles institucionales con atribuciones disímiles en todo el Estado y, adicionalmente, la realidad de un órgano que arbitra mediante criterios puramente jurídicos las potenciales controversias entre aquéllos, el Tribunal Constitucional. Ilustrativamente, véase AJA, Eliseo, El Estado autonómico. Federalismo y hechos diferenciales, Madrid, Alianza, 2003, 357 págs.
} 
federalizable en el ámbito de la configuración territorial del poder ${ }^{3}$. De lo que se desprende, en consecuencia, que con independencia de qué nivel territorial sea el responsable de una competencia concreta, la forma política adoptada, el Estado social, debe impregnar la acción de todas las Administraciones.

No en vano, aquélla «no es sólo una categoría manejable en un contexto político, sino una cláusula constitucional definitoria que prescribe las relaciones entre el proceso social [, y entre éste] y la organización institucional ${ }^{4}$ para lograr hacer universalmente efectivos unos derechos sociales y económicos básicos, "sin los cuales la proclamación de la libertad civil y la libertad política es mera retórica para amplias capas de la población $»^{5}$.

Todo lo cual se traduce, desde el prisma económico-constitucional, en dos consecuencias concatenadas. De un lado, el Estado social se revela como el objetivo último de los ámbitos capitales de la política económica -presupuestario, cambiario, fiscal y monetario- y sus principales instrumentos asociados -niveles de gasto y deuda, masa monetaria, tipo de cambio y tipo de interés ${ }^{6}-$. De otro, la sostenibilidad de la solvencia de las Administraciones Públicas, sobremanera en periodos recesivos con fuertes caídas de la recaudación impositiva, se erige en la máxima garantía del clausulado constitucional, pues con independencia de la fase del ciclo económico por la que transite el Estado; éste, so pena de incumplimiento por omisión, no

${ }^{3}$ Y ello, a pesar de que, si bien la Constitución, en aras del consenso, plasmó la descripción ideal teórica de aquélla como «síntesis entre la firmeza y claridad de sus líneas básicas y la flexibilidad en las modalidades de su actualización» (GARCÍA PELAYO, Manuel, «Consideraciones sobre las cláusulas económicas de la Constitución», en Manuel Ramírez (ed.), Estudios sobre la Constitución española de 1978, Zaragoza, Libros Pórtico, 1979, 488 págs., pág. 29), los dos extremos mencionados, federalización y economía de mercado, aparecían como las soluciones más probables, dadas, respectivamente, tanto la correlación de fuerzas políticas existente como la voluntad de integración en el proyecto de construcción europea, cuyo máximo exponente resultaba el Mercado Común.

${ }^{4}$ SOLOZÁBAL ECHAVARRÍA, Juan José, Tiempo de reformas. El Estado autonómico en cuestión, Biblioteca Nueva, Madrid, 2006, 442 págs., pág. 264.

${ }^{5}$ BEL, Germá, «Desigualdad social, redistribución y Estado de Bienestar», Sistema, N. ${ }^{\circ} 137,1997$, págs. 81-91, pág. 89.

${ }^{6}$ La política comercial y los instrumentos asociados a ella no han sido tomados en consideración dada la previa cesión de soberanía nacional en dicho ámbito. Y ello, por cuanto, de un lado, la adhesión de España al Acuerdo General sobre Aranceles y Comercio (GATT, en su siglas en inglés; por otra parte, antecedente de la OMC) se produjo en una fecha tan temprana como 1963; y, de otro, puesto que la histórica aspiración española de integración en las Comunidades Europeas (con las que se había firmado el Acuerdo Preferencial en 1973), de la que forman parte sus principales socios comerciales, era una realidad. 
puede sustraerse a la imprescindible dotación presupuestaria de recursos suficientes que materialicen los postulados constitucionales.

Así las cosas, los sucesivos embates recesivos experimentados por la economía española desde el ajuste de la Transición hasta el efectuado a resultas de la crisis de 1992 contaban con los instrumentos de política económica referidos. A partir de esa fecha, empero, si bien el crecimiento económico experimentado durante el periodo 1994-2008 difuminaba todo constreñimiento presupuestario en términos de necesidad de minorar los derechos y prestaciones sociales; la firma del Tratado de Maastricht y del Pacto de Estabilidad y Crecimiento en 1997 (y sus revisiones), así como la posterior adopción del euro como moneda oficial de España implicaban, con intensidad creciente en el tiempo, sendas cesiones de soberanía económica. En definitiva, se añadían nuevas limitaciones para la gestión de una crisis económica en el ámbito del Estado social.

Por ello, con el objetivo de presentar las diferencias entre el escenario pre-euro y las implicaciones económico-constitucionales acaecidas fruto de la integración monetaria de España, entre ellas, la reforma de la Constitución de 2011, que se anticipa a las disposiciones del Tratado de Estabilidad, Coordinación y Gobernanza de la Unión Económica y Monetaria de $2012^{7}$; la siguiente sección analiza la convivencia entre las dos crisis económicas más profundas y el Estado social -proyectado y constitucionalizado- con anterioridad a la Moneda Única. Acto seguido, se detalla la presente recesión dentro del contexto euro. Finalmente se refieren algunas propuestas de reforma y se concluye.

\section{CRISIS Y EJERCICIO DE SOBERANÍA ECONÓMICA (1977-1992)}

En pleno proceso de transición hacia un sistema democrático, los datos arrojaban un panorama económico desolador. De manera ilustrativa, a mediados de 1977, y, en términos interanuales, la inflación se situaba en cifras próximas al $30 \%$ y, por ende, «cercanas» a la hiperinflación, y el saldo comercial negativo alcanzaba los 5.300 millones de dólares. La política económica de permisividad del tardofranquismo había agravado las consecuencias de la primera crisis del petróleo (1973) sobre la economía española ${ }^{8}$.

${ }^{7}$ Cuya ratificación se ha autorizado por la Ley Orgánica 3/2012, de 25 de julio.

${ }^{8}$ Paradigmáticamente, SUDRIÁ, Carles, "Crisis económica y transición a la democracia (1975-1985)», ponencia presentada en el Encuentro UIMP «Las 
Así las cosas, el éxito de la Transición política quedaba al albur del resultado de una estabilización económica capaz de salvaguardar la paz social $^{9}$. Ajuste, en consecuencia, orientado a yugular los desequilibrios, tanto interiores como exterior, que motivaban las cifras referidas.

A tal fin, y, con la ventaja técnica que constituía la inexistencia formal y material de un Estado social y sus obligaciones presupuestarias, los Pactos de la Moncloa o Constitución Fiscal extra constitutionem, simultáneamente a la definición de los cimientos para la construcción de una Hacienda Pública dotada suficientes recursos para financiar una suerte de Estado social, o de Bienestar en la terminología anglosajona ${ }^{10}$, pergeñaron las bases para una reactivación del crecimiento desde el ejercicio de la soberanía económica. En este sentido, se impulsó una clara política antiinflacionista (política monetaria restrictiva y reducción de déficit público), acompañada de mecanismos de impulso del sector exterior: devaluación de la peseta, contención pactada del incremento de salarios por debajo de la inflación y medidas liberalizadoras.

Respuesta, en todo caso, similar a la que se ofrecería para combatir los efectos de la grave recesión de 1992-1993 ${ }^{11}$. En el clímax de

catástrofes económicas en la España contemporánea. Las lecciones de la Historia», celebrado en Santander, los días 20 y 21 de agosto de 2012.

A modo de ejemplo, se intentó evitar la imposibilidad de que la vertiginosa escalada de los precios del petróleo impactara en la economía española mediante la absorción del incremento parcial del precio de los derivados del crudo, y todo ello a través de una reducción de la imposición sobre hidrocarburos.

${ }^{9}$ De hecho, en palabras de uno de los partícipes en su redacción, «en el caso del proceso de transición español a la democracia, la elaboración en paralelo de la Constitución política y la fiscal fue, sin duda, una de las claves de su éxito» (BARÓN CRESPO, Enrique, "La Constitución fiscal de 1978», Revista de Occidente, N. ${ }^{\circ} 361$, junio de 2011, págs. 91-101, pág. 101). Idéntico parecer expresó el entonces Ministro de Economía y Vicepresidente Segundo del Gobierno, Enrique Fuentes Quintana (en «De los Pactos de la Moncloa a la entrada en la Comunidad Económica Europea (1977-1986)», Revista ICE, N. ${ }^{\circ} 826,2005$, págs. 39-71, pág. 53).

${ }^{10}$ Ilustrativamente, de acuerdo con los datos de la OCDE, los ingresos fiscales del Estado, que suponían un 18,4\% del PIB en 1975, ascendieron en 2007 a un 35,7 \%. De ahí que el propio Fuentes Quintana sentenciara cómo los Pactos trataban de poner «a la hora de Europa nuestro anticuado cuadro tributario» (Fuentes Quintana, en op. cit. nota 7, pág. 53).

${ }^{11}$ Sin embargo, si bien en el entretanto (concretamente, en 1989) España se había adherido al Sistema Monetario Europeo (SME), lo que inducía una limitación cambiaria-monetaria en la gestión de la crisis económica de principios de los noventa, la virulencia de la misma lo sepultó de facto en 1993. La salida del mismo, entre otros, del Reino Unido e Italia precipitaron la adopción de una banda de cotización cambiaria tan amplia que dilapidó de manera fulminante toda credibilidad en el SME. 
la misma, los datos escenificaban un contexto de intensa caída de la actividad económica: en 1992 se contabilizaron más de 2,5 billones de pesetas de déficit comercial, mientras que en 1993 la deuda sobre PIB superior se situaba por encima del $65 \%$, el déficit público rondaba el $6 \%$ y el paro escalaba vertiginosamente hasta el $25 \%$. Consecuentemente, la contención de precios volvía a erigirse en objetivo principal de un ajuste que se ejecutó, una vez más, con un programa estabilizador canónico: tres devaluaciones consecutivas de la peseta entre septiembre de 1992 y mayo de $1993^{12}$, pacto entre los agentes sociales para lograr un aumento de la masa salarial por debajo del IPC, una política monetaria restrictiva y contención del gasto público.

Dicho lo cual, como contrapunto al contexto de los Pactos de la Moncloa, la «brevedad» de la crisis - cuatro trimestres consecutivos de caída de la actividad-y la capacidad de financiación exterior del déficit presupuestario durante su periodo álgido, lo que, por otra parte, demostraba una confianza en la recuperación de la economía española, permitieron la supervivencia de los programas fundamentales que financiaba el Estado social ${ }^{13}$.

No en vano, si bien los compromisos firmados en Maastricht requerían del cumplimiento de estrictas condiciones de acceso a la futura moneda común ${ }^{14}$, dichas cesiones explícitas de soberanía monetaria, cambiaria, fiscal y presupuestaria deben ser matizadas en dos aspectos fundamentales. De un lado, puesto que, si bien España se adhirió autónomamente a ellos, los compromisos de Maastricht quedaban orientados a aquellos Estados con voluntad de ingresar en

De cualquier manera, la posibilidad de autoexclusión del SME, sin peligro de contagio a terceros, resultaba inmediata, toda vez que la adhesión al mismo se entendía como instrumento de reputación (a semejanza de sistemas como el patrón-oro o Bretton-Woods).

${ }^{12}$ En conjunto, la depreciación de la moneda nacional frente al marco alemán rondó el $25 \%$.

${ }^{13}$ No en vano, y, de acuerdo con los datos de la OCDE, los ingresos fiscales del Estado disminuyeron menos de un $1 \%$ en términos de PIB. Hecho que se compensó, tal y como se ha expuesto, con la activación del resto de los instrumentos de política económica a los que nos venimos refiriendo.

${ }^{14}$ In extenso, el déficit público anual no debía superar 3 por 100 del PIB, el peso de la deuda en el PIB no podía superar el 60 por 100, la tasa de inflación (apreciada por el IPC) no debía resultar superior en 1,5 puntos a la registrada en los tres países europeos con mejor comportamiento en el crecimiento de los precios, los tipos de interés podían fijarse 2 puntos como máximo por encima de los registrados en los países con menor inflación y la unidad monetaria de cada Estado debía mantenerse dentro de los márgenes de fluctuación del SME sin haberla devaluado durante los dos años anteriores a su pretendido ingreso en la Unión Monetaria. 
la futura Eurozona; hecho que permitía descolgarse del acuerdo ${ }^{15}$. De otro, porque la cesión de soberanía monetaria resultaba parcial (las condiciones referidas establecían horquillas), lo que aseguraba un cierto margen de maniobra para la política económica nacional.

Sentado lo anterior, y, a modo de corolario, con anterioridad al contexto de adopción efectiva de la Moneda Única en 1999, el Estado dispuso frente a las dos grandes crisis económicas que se sucedieron entre 1977 y 1992 de suficientes instrumentos asociados a los mecanismos monetarios, presupuestarios, cambiarios y fiscales ${ }^{16}$ para afrontar una caída en la actividad económica que pudiera situar en riesgo los compromisos de gasto adquiridos. Así, la coexistencia de aquellos instrumentos con una progresiva construcción del Estado de Bienestar arrojó un claro balance en términos de prestaciones y derechos sociales: éstos no dejaron de incrementarse a lo largo de la etapa que media entre la democratización de España y la adhesión al euro.

\section{EL EURO Y SU PRIMERA CRISIS: EL NUEVO MARCO DE LA PLAUSIBILIDAD CONSTITUCIONAL}

Merced a las profundas diferencias estructurales y cíclicas ${ }^{17}$ entre las economías de los Estados que han adoptado la Moneda Única, es un lugar común afirmar que la Eurozona no satisface los requisitos que caracterizan un área monetaria óptima, dentro de las cuales las cesiones de soberanía monetaria y cambiaria de sus distintos integrantes quedan empequeñecidas por las ventajas de contar con una moneda única «si los factores [productivos] son móviles a través de

${ }^{15}$ En este orden de cosas, la primera versión del Pacto de Estabilidad y Crecimiento, de obligado cumplimiento para todos los Estados de la Unión Europea, no fue adoptado hasta 1997, en un momento en el que España había iniciado ya tiempo atrás una senda de crecimiento ininterrumpida que se prolongaría hasta el año 2008. De ahí que las limitaciones del propio Pacto (fundamentalmente, dos de las recogidas en Maastricht como condiciones de acceso a la futura moneda única: límites al déficit y a la deuda pública fijados en el 3 y 60 por ciento del PIB, respectivamente) no condicionaran de manera decisiva la política económica del Gobierno, si bien el contenido del acuerdo en sí mismo se ha criticado por su confrontación con los postulados constitucionales, incluso antes de la reforma de 2011 (valga como ejemplo, TORRES LÓPEZ, Juan, «Pactos de Estabilidad y Estado de Bienestar: una nota sobre su dudosa compatibilidad», QPE-Revista Electrónica, núm. 9, enero-abril 2005, págs. 30-40).

${ }^{16}$ Tal y como se ha enumerado anteriormente, fijación de niveles de gasto y deuda, masa monetaria, tipo de cambio y tipo de interés.

${ }^{17}$ Respectivamente, fruto de disimilitudes en modelos de crecimiento, niveles de riqueza..., de un lado; y consecuencia de diferenciales importantes en tasas de inflación, de crecimiento, saldos de la balanza por cuenta corriente..., de otro. 
las fronteras nacionales, [porque] un tipo de cambio flexible ya no es necesario e incluso puede ser claramente perjudicial» ${ }^{18}$. Y es que, a mayor abundamiento, un elevado grado de integración económica «magnifica la ganancia de eficiencia monetaria que consigue el país cuando fija su tipo de cambio frente a las monedas del área, [por lo que] se reduce la pérdida de estabilidad por perturbaciones en el mercado de productos ${ }^{19}$.

En este orden de cosas, a las imprescindibles cesiones de soberanía monetaria y cambiaria inherentes a la adopción de una misma moneda por diferentes Estados, se añadieron, ya desde el Tratado de Maastricht (1992), regulador de las condiciones de acceso al euro, adicionales compromisos fiscales y presupuestarios. Acuerdos reforzados, en este sentido, tanto por el Pacto de Estabilidad y Crecimiento (1997) y sus sucesivas reformas, como por el Tratado de Estabilidad, Coordinación y Gobernanza en la Unión Económica y Monetaria $(2012)^{20}$, en el que se fijan adicionales límites a la deuda y al déficit públicos, así como se disciplinan contundentes mecanismos para su cumplimiento coercitivo. Con todo, las disposiciones fundamentales en materia fiscal-presupuestaria que contiene este último ya habían sido adoptadas por España. De un lado, mediante su intencional esbozo en el nuevo artículo 135 de la Constitución, que remite a la Unión Europea ${ }^{21}$ el establecimiento de las cifras globales anteriormente señaladas; de otro, a través de la promulgación de la Ley Orgánica 2/2012, de 27 de abril, de Estabilidad Presupuestaria y Sostenibilidad Financiera ${ }^{22}$.

De hecho, la reforma constitucional de 2011 y la LO 2/2012 no han acogido sino un endurecimiento sustancial de los requerimientos europeos. Así, mientras la nueva redacción dada al artículo 135 subraya la taxativa obligación por parte de las entidades locales de

${ }^{18}$ MUNDELL, Robert A. (1961). «Una teoría de las áreas monetarias óptimas». American Economic Review, Vol. 51, (págs. 657-665), pág. 664.

${ }^{19}$ KRUGMAN, Paul, y Maurice OBSTFELD (2006). Economía Internacional. Teoría y Política. Madrid: Pearson educación D.L., 742 págs., pág. 612.

${ }^{20}$ Cuyas disposiciones han entrado en vigor el 1 de enero de 2013. España autorizó su ratificación por la Ley Orgánica 3/2012, de 25 de julio.

${ }^{21}$ Irónicamente, el artículo 135 de la Constitución resulta el único del texto que nombra explícitamente a la Unión Europea.

${ }^{22}$ Cuya Disposición Derogatoria Única deroga el marco legislativo anterior, a saber: el Real Decreto Legislativo 2/2007, de 28 de diciembre, por el que se aprueba el texto refundido de la Ley General de Estabilidad Presupuestaria, y la Ley Orgánica 5/2001, de 13 de diciembre, complementaria de la Ley General de Estabilidad Presupuestaria. Legislación, en todo caso, que avaló la Sentencia del Tribunal Constitucional 134/2011, de 20 de julio. 
presentar equilibrio presupuestario (quizá en contradicción con la garantía institucional de la autonomía local) y otorga prioridad absoluta al pago del capital y los intereses de la deuda pública (art. 135.3 CE); la Ley Orgánica que nos ocupa instaura, en su artículo 12, dos reglas de gasto no impelidas por el literal de los textos legislativos de la Unión Europea.

La primera ellas sanciona que la variación del gasto computable de la Administración Central, de las Comunidades Autónomas y de las Corporaciones Locales, no podrá superar la tasa de referencia de crecimiento del Producto Interior Bruto de medio plazo de la economía española (únicamente el desarrollo de reformas estructurales con efectos presupuestarios a largo plazo y, de acuerdo con las previsiones constitucionales, la concurrencia de circunstancias excepcionales podrá amparar una separación de las Administraciones del objetivo de equilibrio o superávit presupuestarios; ex arts. 11.2 y 11.3). La segunda, por su parte, afirma que los ingresos que se obtengan por encima de lo previsto se destinarán íntegramente a reducir el nivel de deuda pública. En consecuencia, si bien ambas disposiciones suponen una apuesta por el equilibrio de las cuentas públicas, éste se predica exclusivamente de los gastos. Nada se dice acerca de la vertiente de los ingresos.

Sentado lo anterior, dentro del contexto euro descrito, para el que se ha llegado a afirmar que el tiempo de los estabilizadores automáticos nacionales ha pasado, resulta pacífico en la literatura económica afirmar que el diseño del euro parece obedecer más a un arquetipo de mera unión cambiaria que a los cimientos de una verdadera unión económica (¿antesala de la homónima política?), pues en origen, y, hasta la fecha, pueden citarse, como principales déficits:

i) el Banco Central Europeo contempla como único objetivo la estabilidad de precios. A diferencia de la Reserva Federal Estadounidense, el crecimiento económico o la función de prestamista de último recurso no se hallan en el texto que informa su estatuto. Esta última función, empero, queda otorgada de facto al Mecanismo Europeo de Estabilidad, tal y como se desprende de su tratado constitutivo, en el que se esbozan múltiples mecanismos de asistencia financiera a los Estados de la Eurozona ${ }^{23}$. De otro lado, únicamente conatos esporádicos y arbitrarios, con sus correlativos costes en tér-

${ }^{23}$ En vigor desde octubre de 2012, sus artículos 14 a 18 instrumentan diferentes procedimientos de apoyo a la estabilidad: líneas de crédito, recapitalización de entidades financieras, préstamos directos e intervenciones en los mercados primario y secundario de deuda pública. 
minos de incertidumbre financiera para el mercado y de reputación para la propia institución, han jalonado el ejercicio de la función de prestamista de último recurso de los Estados de la Eurozona por parte del BCE.

ii) no existe una unión bancaria. Cuando se escriben estas líneas, si bien resulta una ambición que se encuentra en la agenda europea, la supervisión del sector financiero se mantiene descentralizada $^{24}$.

iii) la Eurozona (al igual que la Unión Europea en su conjunto) carece de recursos suficientes para desarrollar políticas de estabilización fiscal/presupuestaria que palien el círculo vicioso austeridadrecesión que acontece en numerosos Estados Miembros, dados los objetivos de déficit fijados para éstos, sin amenazar los propios compromisos de gasto de sus Estados sociales respectivos.

Por todo ello, la implementación de un programa estabilizador que estimule el retorno a la senda del crecimiento económico tras un periodo recesivo, en el que se produce tanto un incremento de gastos públicos coyunturales (intereses de la deuda, seguros de desempleo...) como una rigidez a la baja de muchos de los estructurales (especial relevancia cobra la provisión de bienes públicos preferentes por parte del Estado ${ }^{25}$ ), queda enormemente dificultado, de un lado, por los dos tipos de divergencias entre economías mencionados con anterioridad, que coadyuvan al contagio patológico en el medio plazo de todas ellas; de otro, por cuanto el nuevo marco normativo sitúa la plausibilidad constitucional anclada a una disciplina fiscal, obligación suprema de todos los Estados. Los cuales, simultánea-

${ }^{24}$ Únicamente para aquellas entidades que hayan recibido fondos del Mecanismo de Estabilidad su supervisión la ejercerá el BCE a partir del 1 de enero de 2013. La supervisión única parece que no se establecerá hasta 2014, y, además de para las anteriores, solo para aquellas entidades que representen un riesgo sistémico

${ }^{25}$ Aquéllos «de naturaleza privada que el Sector Público subvenciona o provee gratuitamente por considerarlos esenciales para el desarrollo de una vida digna» (ALBI, Emilio, GONZÁLEZ PÁRAMO, José Manuel e Ignacio ZUBIRI, Economía Pública I, Barcelona, Ariel Economía, 2009, 515 págs., pág. 315). Así, se trata de bienes y servicios (i) cuya disponibilidad para el sujeto no trae causa de sus propias elecciones individuales, pero (ii) cuyo consumo contiene tanto efectos privados como externos y que, adicionalmente, (iii) poseen un valor sociopolítico fundamental al cumplir con objetivos relacionados con la equidad como el aseguramiento de la igualdad de oportunidades y el logro de metas redistributivas; lo que hace que (iv) su financiación suponga la mayor porción de los presupuestos públicos en los países desarrollados (tal y como se desprende de los datos publicados por la OCDE; en los que en el indicador medio para la OCDE29 en 2008 muestra cómo la financiación de los bienes preferentes alcanza casi un 30\% del PIB y supone una cuantía dos veces superior a la de los bienes públicos); principalmente, Sanidad, Educación y Vivienda. 
mente, carecen del resto de instrumentos de política económica para cohonestar la tríada Estado social, recesión económica y observancia de los compromisos europeos, en su redacción actual.

De ahí que, en consecuencia, el estancamiento prolongado de la economía española ${ }^{26}$ (a pesar de la pujanza del sector exterior, éste no alcanza un quinto del PIB), junto a los actuales compromisos de déficit asumidos, en contra de los cuales se sitúan las recomendaciones de instituciones nada sospechosas de intervencionistas como el Fondo Monetario Internacional ${ }^{27}$, no pueda ser autónomamente asumido sin drásticas reducciones de los niveles de gasto público, que, a su vez, acentúan la depresión en coyunturas de hundimiento del consumo y la inversión internos. Máxime cuando, de una parte, los programas de consolidación presupuestaria en recesión, a tenor de las recientes y exitosas experiencias históricas sueca y finlandesa de los años noventa, se rigen por pautas muy bien definidas ${ }^{28} ; \mathrm{y}$, de otra, por cuanto la minoración de los gastos públicos se está ejecutando de manera lineal29.

Así las cosas, en el mes de julio de 2012 España ha solicitado la intervención europea para la recapitalización de su sector financiero.

\section{CONCLUSIONES: ¿SOBERANOS O INTERVENIDOS?}

El crecimiento económico y unas finanzas públicas robustas y saneadas, objetivo al que sirve la instauración de reglas fiscales, constituyen la condición indispensable para una plausible dotación de recursos económicos que, de forma efectiva, pueda materializar los postulados constitucionales. Por ello, y, para salvaguardar tanto la Ha-

${ }^{26}$ Dos recesiones en menos de tres años (2008-2011) con un bagaje extremadamente nocivo: desplome de los ingresos fiscales, una tasa de paro entorno al $25 \%$, un déficit con improbables posibilidades de reducirse hasta el $3 \%$ del PIB en 2013, necesidades de financiación exterior superiores al $4 \%$ del PIB...

${ }^{27}$ Valga como ilustración su influyente documento de 2009, «Fiscal RulesAnchoring Expectations for Sustainable Public Finances»,

http://www.imf.org/external/np/pp/eng/2009/121609.pdf(03/09/2012)

${ }^{28}$ En síntesis, el desapalancamiento privado y público debe discurrir por sendas diametralmente opuestas (valga como ilustración MCKINSEY GLOBAL INSTITUTE, «Debt and Develeraging: Uneven progress on the path to growth», 2012,

http://www.mckinsey.com/insights/mgi/research/financial_markets/uneven_ progress_on_the_path_to_growth), 03/09/2012

${ }^{29}$ Valga como ejemplo, SERRANO SANZ, José María, «La primera crisis de la España comunitaria (1992-1993)», ponencia presentada en el Encuentro UIMP «Las catástrofes económicas en la España contemporánea. Las lecciones de la Historia», celebrado en Santander, los días 20 y 21 de agosto de 2012. 
cienda como los programas públicos del Estado social que la primera financia, los sucesivos gobiernos de España utilizaron, en el combate de las dos grandes crisis descritas previas a la adopción del euro, todos los resortes de los que disponía la política económica nacional.

En este orden de cosas, y, a partir de 1994, si bien la senda de crecimiento que se había iniciado difuminó las progresivas cesiones de soberanía económica a las que, en pos de la integración en la Moneda Única, se adhirió España; aquéllas implicaban el traslado del control de los principales instrumentos de toda política de estabilización a las instancias comunitarias: fijación de los niveles de deuda y déficit, tipo de cambio y tipo de interés. Así, mientras las herramientas monetarias han quedado absorbidas por el Banco Central Europeo; el incumplimiento de los dos primeros, cuya fijación corresponde a la Comisión Europea, ha quedado fuertemente dificultado, so pena de importantes sanciones, la más importante de las cuales resultará el estrangulamiento de la financiación mediante una negativa a la actuación del Mecanismo Europeo de Estabilidad.

Con todo, los cauces de estabilización europeos vigentes quedan destinados, de manera exclusiva, a facilitar una mejora en las condiciones de financiación de los Estados que insten la intervención del Mecanismo de Estabilidad para acudir en su rescate ${ }^{30}$. Por tanto, no existe instrumento alguno cuyo objetivo tienda a estimular el retorno a la senda del crecimiento económico del país de que se trate en escenarios azotados por la trampa de la liquidez o inmersos en fortísimos procesos de desapalancamiento público-privados.

En consecuencia, ya en clave española, si continúa el desplome de los ingresos públicos y del consumo interno sin una variación del marco actual, la única vía para el cumplimiento progresivo de los objetivos presupuestarios pasa, bien por la entrada a un nuevo escenario en el que los gastos igualen a los ingresos en flexibilidad, bien a otro en el que estos últimos se equilibren rígidamente a los gastos. Máxime cuando, al calor de los compromisos europeos firmados, la reforma constitucional de 2011 instituye el equilibrio presupuestario como la columna vertebral del armazón constitucional en su conjunto.

Lo que se traduce, en definitiva, en un marco constitucional cuya provisionalidad, fruto de la pérdida de soberanía económica, ya no se encuentra en la voluntad nacional, sino europea. Por todo ello, la mejora de la compatibilidad entre superación de crisis económicas

${ }^{30}$ La actuación del BCE como prestamista de último recurso persigue el mismo fin citado. 
y Estado social podría beneficiarse de la implementación conjunta de nuevas iniciativas europeas y nacionales, pues la experiencia histórica, sobremanera la de la última década, informa de una característica geográfica-temporal acerca del fenómeno de las depresiones económicas: su desigual incidencia coyuntural, pero la inexistencia de inmunidad frente a ellas.

A tal fin, y siempre con el objetivo de apostar por un proceso de integración europea comprometido con el Estado social (después de todo, la única institución «que ha permitido que las sociedades europeas consigan unos niveles de riqueza y cohesión e integración social sin parangón histórico ${ }^{31}$, valores cruciales para una convivencia ordenada), se defiende la creación de mecanismos europeos de estabilización, premisa de crecimiento, frente a situaciones de depresiones económicas, toda vez que la soberanía sobre los instrumentos de política económica más decisivos ya no reside en los diferentes Estados que conforman la Eurozona. Así, se comparten posiciones que abogan por i) la fijación de objetivos de déficit realistas con la evolución de las cifras de crecimiento económico de cada Estado, ii) una definición clara del papel del Banco Central Europeo que evite un estigma de arbitrariedad, iii) un incremento sustancial en el presupuesto europeo que posibilite su actuación como instrumento de estabilización en la Eurozona, iv) el alumbramiento de mecanismos de mutualización de deuda pública, de acuerdo con algunos umbrales y condiciones ${ }^{32}$, v) la mejora de la integración/armonización fiscal, y vi) la introducción, frente a los actuales e insostenibles en el largo plazo desequilibrios comerciales, de mecanismos de solidaridad europea, en coexistencia con la política de cohesión y ligados a los saldos comerciales bilaterales ${ }^{33}$.

En todo caso, dicho programa debe complementarse con uno homónimo a escala nacional, de acuerdo con la arquitectura institucional-constitucional de cada Estado de la Eurozona, orientado a potenciar la eficiencia y equidad en la intervención del Sector Público. Desde el prisma español, se defienden dos iniciativas principales. En primer lugar, se ampara una clarificación del Estado social. Para ello se debería apostar, de un lado, por la elaboración de un presupuesto

${ }^{31}$ BEL, loc. cit. pág. 2 nota 5, pág. 82 .

${ }^{32}$ Valgan como ejemplo los esbozados en el informe "Completing the Euro", elaborado por el «Grupo Tommaso Padoa-Schioppa», 2012,

http://www.notre-europe.eu/uploads/tx_publication/CompletingTheEuro_ReportPadoa-SchioppaGroup_NE_June2012.pdf (03/09/2012)

${ }^{33}$ En SANZ ARCEGA, Eduardo, «Crisis y Estado de Bienestar: ¿riesgo u oportunidad para España?», inédito 
partida a partida, sin reducciones lineales y mediante una definición de las prioridades que aúne el mayor consenso parlamentario y social posible; y de otro, desde el punto de vista de los ingresos, mediante una reforma integral del sistema fiscal y de la inspección tributaria que potencien elementos de equidad ${ }^{34}$.

En segundo término, se defiende la modificación de los sistemas de financiación de las Comunidades Autónomas y de los Entes Locales, de modo que aquellas jurisdicciones con una presión fiscal menor no reciban transferencias de otras que ostenten unos niveles mayores. De manera adicional, y, por la importancia que, en términos presupuestarios, revisten las Comunidades Autónomas, sus prioridades de gasto deben dirigirse a la financiación de los bienes y servicios públicos preferentes; para lo cual la definición de umbrales y estándares de calidad deviene en imprescindible (tanto por lo que respecta al aspecto de la financiación en sí mismo como en lo referente al aseguramiento de unos mínimos vitales comunes para todos los españoles).

Mediante las propuestas explicitadas, si bien no podrá hallarse entre ellas un Bálsamo de Fierabrás, quizá, en conjunto, sí puedan contribuir a la minoración, en contextos de crisis económicas, del elemento de provisionalidad del que gozan en la actualidad los marcos constitucionales de los países que conforman la Eurozona. Uno de cuyos máximos exponentes, el Estado social, resulta la única fórmula política que ha logrado conjugar democracia, mayor esperanza de vida de los ciudadanos y prosperidad ${ }^{35}$.

${ }^{34}$ No en vano, la literatura económica pone de manifiesto cómo la percepción de justicia del sistema fiscal condiciona directamente el nivel de cumplimiento de los ciudadanos.

${ }^{35}$ LINDERT, P. H. (2004). Growing public. Social Spending and Economic Growth Since the Eighteenth Century. Cambrigde, Cambridge University Press, 337 págs. 